\title{
PRINCIPLES AND PROBLEMS OF CLINICAL TRIALS ILLUSTRATED BY FINDINGS FROM THE UNIVERSITY GROUP DIABETES PROGRAM AND THE CORONARY DRUG PROJECT
}

\author{
Christian R. Klimt, M.D., Dr. P.H.* \\ and \\ Suketami Tominaga, M.D., M.P.H.**
}

The need for the quantitative evaluation of benefit and risk of new therapeutic measures-initially drugs but more and more so also surgery, radiotherapy, and medical devices-has come to the fore since the passage of the Harris-Kefauver law in 1962 . In the USA the Food and Drug Administration (FDA) requires a drug company to prove the efficacy and safety of a new drug by conducting three phases of clinical trials before a prescription drug is licensed and sold (Figure 1).

\section{FDA CLASSIFICATION OF CLINICAL DRUG TRIALS}

A phase I clinical trial is to study: absorption, excretion, and short-term toxicity of a new drug and to determine a safe dosage range on a relatively small number of healthy men, while a phase II trial is to study without controls the efficacy and safety of the new drug on a relatively small number of patients. Phase III and phase IV trials are both controlled clinical trials on a relatively large number of patients, but there is an important difference between the two types of trial. In the phase III clinical trial the endpoint is either chemical or physical, such as blood glucose lowering effect or blood pressure lowering effect, as well as quantitation of adverse effects. In the phase IV type clinical trial the endpoint is clinical, such as mortality, morbidity, and quality of life.

Phase IV type clinical trials are not mandatory before a new investigational drug is licensed and sold, but the FDA requires the drug company to study and report the long-term efficacy and safety of the new drug after it is licensed and marketed.

* Dr. Klimt is Professor and Director, and **Dr. Tominaga is Associate Professor of University of Maryland, School of Medicine, Department of Social and Preventive Medicine, Division of Clinical Investigation.

Reprint requests: Dr. Christian R. Klimt Univrsity of Maryland, Division of Clinical Investigation 610 W. Lombard Street, Baltimore, Maryland 21201, U.S.A. 


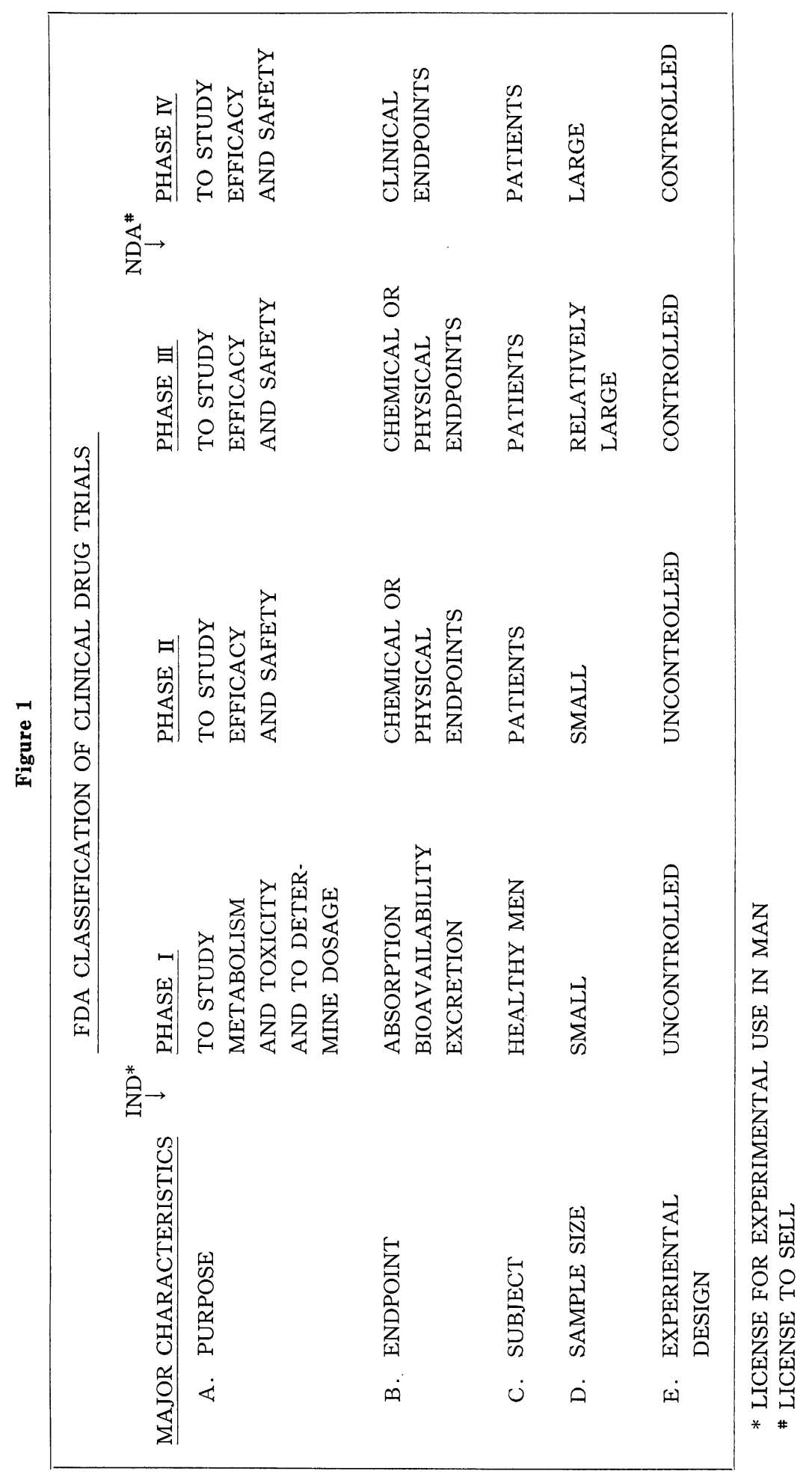


When a new drug is licensed by the FDA and becomes available on the market, how might the physician react?

Let me borrow a quotation ascribed to Sir William Osler advising his young fellows in medicine. "Go out and use a new treatment as quickly as possible"; he advises, "use it as long as it still remains useful." A lot is implied in this quotation. Basically the activism of the physician, his optimism in being of help to the patients, his readiness to accept somthing new, and often his lack of patience to wait for appropriate confirming data before he starts a treatment without surely knowing what will result in the long run. This is particularly dangerous in the case of long-term treatment since very few, if any, medication fails to produce toxic reactions, if given day in and day out to the chronically ill patient. More likely than not, if the patient survives, there will be a time when he suffers more from side reactions than gains benefit from continuous treatment. That is why the phase IV type clinical trial is necessary to test the long-term efficacy and side effects of the drugs for chronic diseases. I would like to devote this lecture to the principles and problems of the phase IV clinical trial.

\section{EXAMPLES OF THE PHASE IV TYPE CLINICAL TRIALS}

The University Group Diabetes Program (UGDP) ${ }^{1-4)}$ and the Coronary Drug Project $(\mathrm{CDP})^{5-7)}$ are considered typical examples of the phase IV clinical trial because in the UGDP :

the main purpose of the study is to evaluate the efficacy of several hypoglycemic agents in reducing mortality and cardiovascular complications in patients with mild adultonset diabetes mellitus,

and in the CDP :

the main purpose is to evaluate the efficacy of several blood lipid lowering drugs in reducing mortality and morbidity in patients with coronary heart disease.

\section{MAJOR FEATURES AND PROBLEMS OE THE PHASE IV TYPE CLINICAL TRIAL}

If you want to conduct a phase IV type clinical trial, you may encounter some other difficulties which you usually do not have in the phase II or III type study. I will illustrate several important features and problems using the UGDP and CDP data (Figure 2).

Two main factors with respect to the scale of the phase IV trial are the sample size and the follow-up period. Usually, a phase IV type trial to test the efficacy of a drug for chronic disease requires a large sample size and a long follow-up period.

Why is a large sample size required in the phase IV trial? There are two main reasons: (1) the frequency of the events of interest, e.g., death and coronary heart attacks, is relatively low; (2) the effect of a drug against a chronic disease may not be as dramatic as that of antibiotic treatment for acute infectious diseases. These two factors contribute to a need for a large sample size. 
Figure 2

\begin{tabular}{|l|}
\hline MAJOR FEATURES AND PROBLEMS OF THE \\
PHASE IV TYPE CLINICAL TRIAL \\
\hline - SCALE OF THE TRIAL \\
SAMPLE SIZE \\
FOLLOW-UP PERIOD \\
- NEEDS OF MULTICLINIC COOPERATIVE \\
STUDY \\
- COMMON PROTOCOL AND CENTRAL \\
DATA COLLECTION AND PROCESSING \\
- METHODS OF EFFICACY AND SAFETY \\
MONITORING \\
-STATISTICAL PROBLEMS \\
-COST
\end{tabular}

\section{SAMPLE SIZE DETERMINATION IN THE CDP}

An example of the sample size required in a phase IV type clinical trial is given in Figure 3. In the Coronary Drug Project we wanted to evaluate five active lipid influencing treatments using one common placebo treatment. The assumptions used are:

Five year mortality of $30 \%$ for the placebo treated group and $22.5 \%$ in each of the five active drug treatments. Namely we expected to test for a $25 \%$ reduction in mortality by active drug treatments. The expected five year dropout rate was estimated as $30 \%$ and an alpha-level of 0.01 and a beta-level of 0.05 were used.

Thes, it turned out that we needed a total of about 8,300 patients ; about 1,000 patients for each of five active drug treatments and about 2,800 patients for the placebo group.

Figure 3

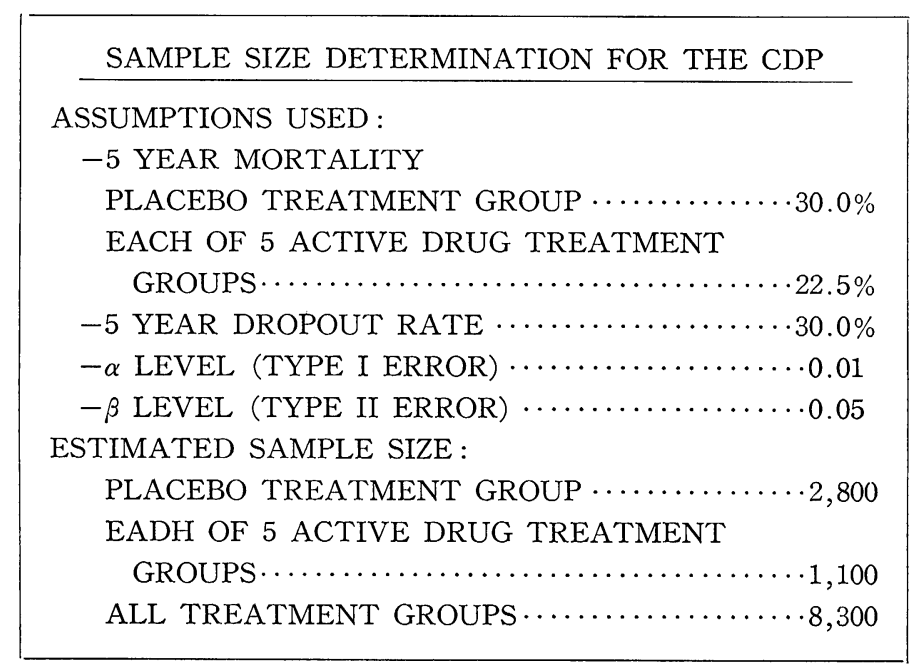


Actually, 8,341 patients were recruited in the Coronary Drug Project.

You may wonder why the sample size for placebo group is larger than that for each of the five active treatments. Since each of the drug tested groups is to be compared with the same placebo group, it is necessary that the placebo death rate be measured with greater precision than any of the drug death rates. Using a procedure which minimizes the variance of the observed difference between the five year death rate for a drug group and that for the placebo group, it was determined that the size of the placebo group should be approximately 2.5 times as large as that of any one of treatment groups. More detailed methods of the sample size determination used in the CDP have been published elsewhere ${ }^{5}$.

Why is a long follow-up period needed? There are several reasons:

A. to study long-term efficacy of drugs for chronic diseases.

B. to study long-term safety and toxicity of drugs for chronic diseases.

C. to study the natural history of the chronic disease of interest using the placebotreated patients.

\section{MULTI-CLINIC COOPERATIVE STUDY}

Another common characteristic of the phase IV type study is the need for a "Multiclinic cooperative study". As one would imagine from the large sample size which is a usual requirement of the phase IV study, it seems almost impossible to recruit that large a number of patients from a single hospital. Therefore, it is necessary to recruit a sufficient number of study subjects through many clinics. One important advantage of a multi-clinic cooperative study is better representation of different clinical practice, locatity, sex, race, and other socioeconomic characteristics. On the other hand, standardization of procedures is much more difficult in this type of study.

Besides many clinical centers several special centers are necessary such as a Coordinating Center, Laboratory Center, ECG Reading Center, and Drug Procurement and Distribution Center, etc. A multi-clinic cooperative study requires also the cooperation of various specialists; clinicians, pharmacologists, epidemiologists, statisticians and others (Figure 4).

There are some requirements and problems which are specific to a multi-clinic cooperative study. First of all, it is necessary to use a detailed common protocol among all participating clinics so that the data can be pooled. Otherwise, it is difficult to analyze the data and to draw valid conclusions. "To use the same criteria and same method" does not guarantee the same results. Clinics will vary in many ways and chance variation of findings must be considered. It is also desirable to collect, process and analyze data centrally. Urine or serum specimens may be sent to the laboratory center and the results are forwarded to the Coordinating Canter. ECG's, X-ray's or fundus photos may be interpreted at special centers, preferably by the same reader or readers. Data are then forwarded to the Coordinating Center. There, all data are collected, processed and analyzed. It is usually a responsibility of the Coordinating Center to prepare reports for the study group, various committees or for publication. Authorship of reports or papers belongs to all participating investigators. 
Figure 4

\begin{tabular}{|l|}
\hline \multicolumn{1}{c|}{ COOPERATIVE STUDY } \\
COOPERATION AMONG ORGANIZATIONS : \\
-CLINICAL CENTERS \\
- COORDINATING CENTER \\
-LABORATORY CENTER \\
-ECG CENTER \\
-DRUG DISTRIBUTTION CENTER \\
COOPERATION AMONG SPECIALISTS : \\
-CLINICIAN \\
-PHARMACOLOGIST \\
-STATISTICIAN \\
-EPIDEMIOLOGIST \\
-OTHER SPECIALISTS \\
\hline
\end{tabular}

\section{BASIC PRINCIPLES AND METHOD OF THE CLINICAL TRIAL}

There are several basic principles or methods which are used in both phase III and IV type drug trials (Figure 5):

A group of suitable patients is being randomly allocated to one of several treatment groups including a control group. The control group can either be placebo or a standard accepted treatment. Emphasis lies on randomization, implying that the assignment to treatment group has a defined probability as every patient is assigned and included into the study. Other features of the classical trial are an attempt at double-blindness, implying teat neither the patient nor the treating physician is aware of the medication given. This becomes less imperative if the endpoints, on the basis of which the trial is decided, can be determined objectively and preferably outside the immediate clinical environment in which patients are treated. Examples would be results from X-rays, electrocardiograms, encephalograms, fundus photographs, and the like.

Figure 5

\begin{tabular}{l} 
BASIC PRINCIPLES AND METHODS \\
IN THE CLINICAL DRUG TRIAL \\
\hline -RANDOM ALLOCATION \\
-CONTROL GROUP \\
-DOUBLE-BLINDNESS
\end{tabular}

In reality, none of these basic methods is easy to adopt or maintain, but all of them are essential design principles in any phase III or IV type clinical trial. 


\section{REQUIREMENTS AND PROBLEMS OF A PHASE IV TYPE TRIAL}

Besides basic principles or methods which are commonly used in the phase II or III type study there are several important requirements or problems associated with a long-term phase IV type trial.

- continuouous efficacy and safety monitoring

- violations of usual treatment methods,

-statistical problems,

- cost, etc.

\section{CONTINUOUS EFFICACY AND SAFETY MONITORING}

In a long-term clinical trial it is necessary in order to protect the participating patients to evaluate the efficacy and safety of treatments periodically. The purpose of repeat data evaluation is to detect either beneficial or untoward effects of treatments as soon as possible and to act accordingly. Because of undesirable effects some treatments in the UGDP and CDP were prematurely discontinued ${ }^{1,3,6,7)}$.

\section{VIOLATIONS OF USUAL TREATMNT METHODS}

In order to maintain double-blindness and to assure continuous comparability of treated groups with the control group, the investigators have to settle on a standard treatment regimen. This may be either a constant standard dose so selected that it is neither homeopathic nor likely to be toxic or else codifying the rules for treatment which have to be strictly adhered to in individual patients. The latter path is often very difficult to establish to everyone's satisfaction.

If a fixed dose of a drug is given to a patient for a long period, treatment failures, either primary or secondary, may be encountered. Usually, it is not permissible to switch one drug to another when a treatment failure occurs and it is also discouraged to use other drugs which may confound the results of the study drugs. For example, in the CDP where the main purpose is to test the efficacy of several cholesterol lowering drugs, other lipid lowering therapy excludes a patient at the outset. Practicing physicians often claim that this way of treatment is a violation of the usual way in which they treat patients, thus, the results obtained from the clinical trials such as the UGDP are in their eyes not applicable to the usual personalized treatment of patients by practicing physicians.

It may be possible to simulate the physician's usual treatment method in the clinical trial without jeopardizing double-blindness, but the treatment protocol would become quite complex and the analysis of data would be extremely complicated.

Let me point out one thing. There is a tendency among physicians to resist acceptance of unexpected results, especially when negative results are obtained and they try to find explanations of the negative results in the methods of the trial rather than in the treatment. I wonder if physicians might not have been quiet about the use of a fixed dose of oral 
hypoglycemics in the UGDP if positive results had been obtained. The UGDP was not primarily designed to test toxicity but to demonstrate possible beneficial effects of phyoglycemic agents with respect to mortality and vascular complications in non-insulin dependent diabe$\operatorname{tics}^{1)}$.

\section{STATISTICAL PROBLEMS}

To the uninitiated the analysis of the results from clinical trials is a straightforward statistical matter for which textbook statistics suffice. In reality we are a long way from having a standard acceptable battery of analyses which is capable of dealing with all the problems inherent in long-term clinical trials (Figure 6). Just to mention a few of the

Figure 6

\begin{tabular}{|c|c|}
\hline \multicolumn{2}{|c|}{ STATISTICAL METHODS AND PROBLEMS } \\
\hline -REPEAT DATA EVALUATION, & RELATIVE BETTING ODDS (RBOs) \\
\hline -MULTIPLE TREATMENTS $\}$ & MONTE CARLO CONTROL CHART \\
\hline -VARIABLE FOLLOW-UP PERIOI & LIFETABLE METHOD \\
\hline $\left.\begin{array}{l}\text {-MULTIPLE RISK FACTORS } \\
\text {-MULTIPLE ENDPOINTS }\end{array}\right\}$ & MULTIVARIATE ANALYSIS \\
\hline -COMBINATION OF ABOVE.. & $\begin{array}{l}\text { NO SATISFACTORY ANALYTIC } \\
\text { TECHNIQUE YET AVAILABLE }\end{array}$ \\
\hline
\end{tabular}

difficultes of classical analytic methods. Most studies are designed on the principle of fixed sample size, fixed duration. In reality long-term trials require analyses in the course of the study for the reasons I already mentioned.

Besides the repeat data evaluation, more than one active drug may be tested using one common placebo treatment, namely, "multiple treatments". Under these circumstances ciassical statistical tests such as chi-square test or the Student's t-test are inadequate.

In a large-scale study patients must be recruited over a long period. This implies that the follow-up period of patients may vary from case to case. To solve this problem it is necessary to use a life table method or cohort analysis.

To pay the appropriate statistical price for these repeated analyses and multiple comparisons several techniques have been evolved. They include the method of relative betting odds (RBO's) developed by Jerome Cornfield ${ }^{8)}$ and and adaptation of Monte Carlo techniques which simulates trials on computers using appropriate models such as life tables. The latter method has been modified by one of our statisticians, Dr. Paul Canner, so that it is usable in a clinical trial ${ }^{9)}$ Both of these methods have as yet not advanced to the stage where they can correct for a second type of error; namely, the choice of one or several response variables out of a multitude available to apply statistical tests to. For these selections again an appropriate price has to be paid, but we do not know as yet how to do that. Especially difficult is the situation where the same drug has both beneficial and adverse effects. 
In a study of chronic disease usually many risk factors are involved. In the CDP we have well over 100 baseline characteristics including 10 to 20 important risk factors. To solve this problem we must use some sophisticated multivariate analytical methods.

Unfortunately, to date no ideal statistical technique is available which considers all problems simultaneously.

I will show some examples of the statistical analysis and the decision-making process based on our experience in the UGDP and CDP.

\section{EXPERIENCE IN THE UGDP}

In the UGDP there were five treatment groups including a placebo treatment and three hypoglycemic agents; insulin, tolbutamide and phenformin (Figure 7). There are two treatment groups for insulin; in one treatment called "Insulin Variable" as much insulin as is required to maintain "normal" blood glucose has been used and in another insulin group called "Insulin Standard" 10 to 16 units per day of U.80 Lente insulin has been used depending on a patient's body surface. Fixed daily deses of tolbutamide and phenformin were given. Approximately 200 patients were assigned to each treatment group. A standard prescription of diet therapy has been given to all patients across the board.

Figure 8 shows life table cumulative mortality rates for all causes (part A) and for cardiovascular causes (part B). Beginning 3 years after the initiation of treatment, the tolbutamide-treated group showed excess overall mortality, especially from cardiovascular causes. The TOLB-PLBO difference became statistically significant and the tolbutamide treatment was discontinued in $1969^{1)}$. Figure 8 also shows that, as far as mortality is concerned,

Figure 7

\begin{tabular}{|c|c|c|}
\hline \multicolumn{3}{|c|}{$\begin{array}{c}\text { STUDY TREATMENTS AND NUMBER OF PATIENTS ASSIGNED } \\
\text { TO EACH TREATMENT IN THE UGDP }\end{array}$} \\
\hline TREATMENT (ABBREVIATION) & DOSAGE PER DAY & NO. OF PATIENTS \\
\hline INSULIN VARIABLE (IVAR) & $\begin{array}{l}\text { AS MUCH INSULIN } \\
\text { AS IS REQUIRED } \\
\text { TO MAINTAIN } \\
\text { "NORMAL" BLOOD } \\
\text { GLUCOSE }\end{array}$ & 204 \\
\hline INSULIN STANDARD (ISTD) & $\begin{array}{l}\text { 10-16 UNITS } \\
\text { DEPENDING ON } \\
\text { PATIENTS' BODY } \\
\text { SURFACE }\end{array}$ & 210 \\
\hline TOLBUTAMIDE (TOLB) & $1.5 \mathrm{gm}$ & 204 \\
\hline PHENFORMIN (PHEN) & $100 \mathrm{gm}$ & 204 \\
\hline LACTOSE PLACEBO (PLBO) & & 205 \\
\hline & TOTAL & 1,027 \\
\hline
\end{tabular}


Figure 8

CUMULATIVE MORTALITY RATES PER 100 POPULATION AT RISK

BY YEAR OF FOLLOW-UP UGDP, OCTOBER 1969
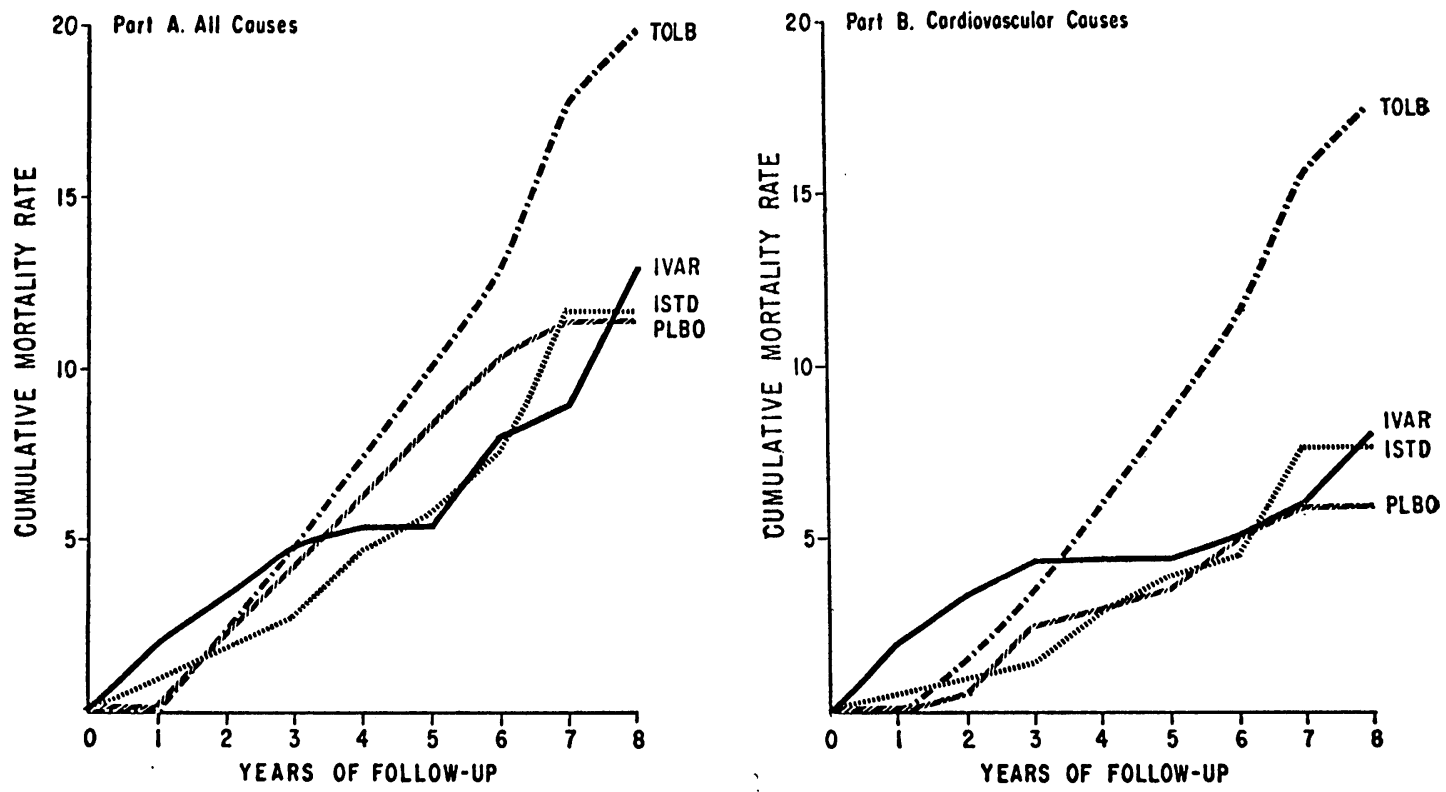

UGDP (1970)

the combination of insulin and diet therapy is no more effective than diet therapy alone.

Figure 9 shows Monte-Carlo five percent monitoring boundaries for differences in cumulative number of deaths. Tolbutamide-placebo difference in cardiovascular deaths (Part B) crossed the upper boundary sometime in 1967. Crossing the upper boundary means tolbutamide treatment shows higher mortality from cardiovascular causes than placebo treatment at a significance level of $5 \%$ after taking into account repeated data evalusation over the projected length of the study.

In spite of the random allocation of patients to the treatment groups, there were some imbalances between the two treatment groups with respect to several baseline characteristics. In order to study whether or not the imbalance contributed to the excess mortality in the TOLB group, various statistical analyses were made. Figure 10 shows the way of simultaneously adjusting for the imbalance of various baseline characteristics using the logistic model. White bars in Figure 10 show actual observed mortality rates and shaded bars show estimated or adjusted mortality rates after taking into account 13 major baseline characteristics simultaneously. The slightly higher estimated mortality rate from cardiovascular causes in TOLB group compared to the observed rate suggests the TOLB-PLBO difference in cardiovascular death became even larger when the imbalance of the 13 major baseline characteristics were adjusted simultaneously.

In the case of tolbutamide treatment there was no clear-cut clue to explain the mechanism of excess mortality. Some investigators have reported that tolbutamide has inotropic 
Figure 9

MONTE CARLO FIVE PERCENT MONITORING BOUNDARIES FOR DIFFERENCES IN CUMULATIVE NUMBER OF DEATHS (CENTER LINE IS THE MEAN DIFFERENCE IN NUMBER OF DEATHS FROM ALL SIMULATED REPLICATIONS OF THE TRIAL)

TOLBUTAMIDE VERSUS PLACEBO

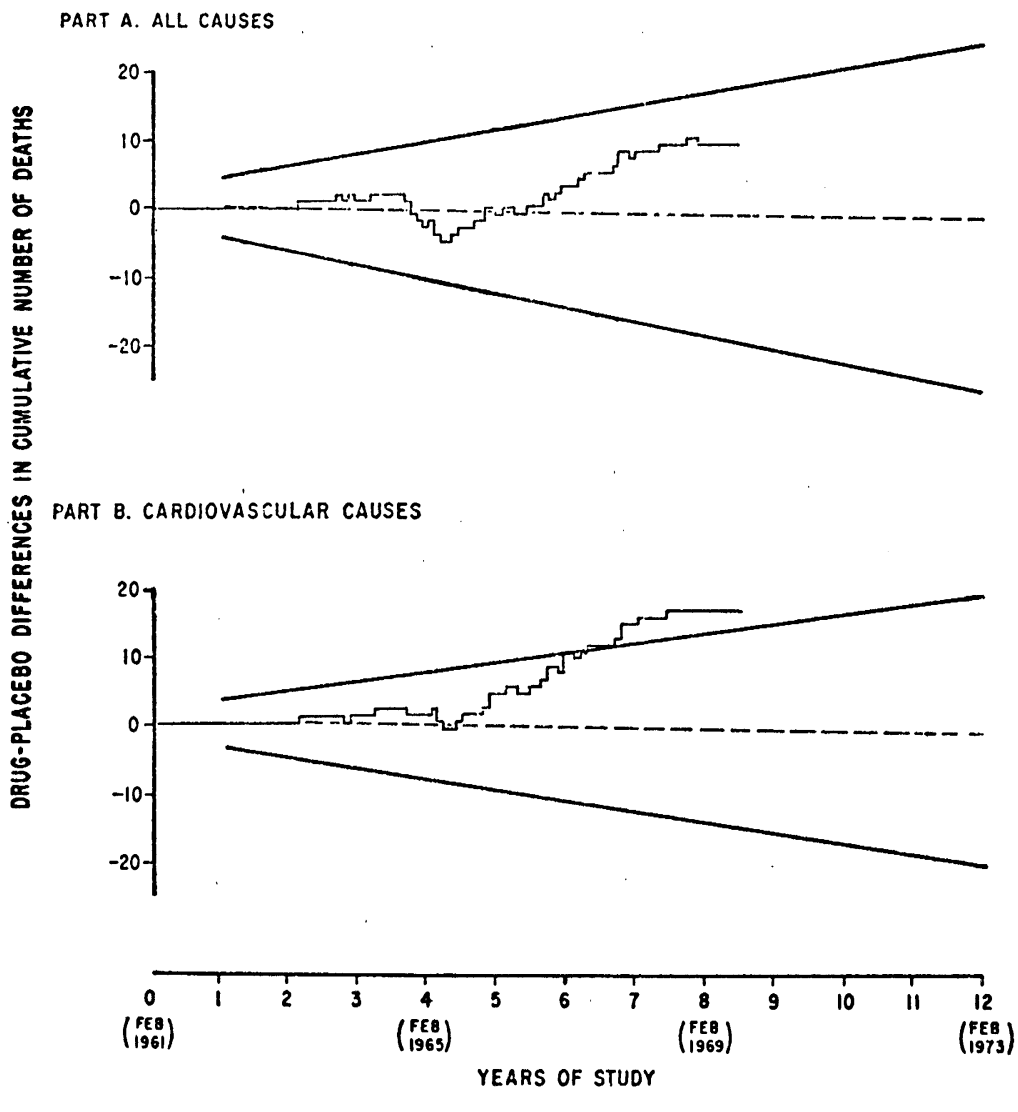

$\operatorname{UGDP}(1970)$

effects on the myocardium ${ }^{11}$. Others have observed hypokalemia and effects on atrio-ventricular conduction ${ }^{12)}$.

Phenformin treatment was added to the original treatments in 1962 because of its different mode of action in lowering blood glucose levels. In 1971 this treatment was also discontinued because of excess mortality, particularly from cardiovascular causes (Figure 11) ${ }^{3)}$. In contrast to the tolbutamide effects where the excess mortality became apparent more than three years after the initiation of treatment, phenformin treatment showed similar effects already during the second year. We have some clues why phenformin treatment may have caused excess cardiovascular deaths. There was evidence that phenformin treatment elevated 
Figure 10

OBSERVED AND ESTIMATED MORTALITY FROM

THE MULTIPLE LOGISTIC MODEL UGDP, OCTOBER 1969

PART A. ALL CAUSES

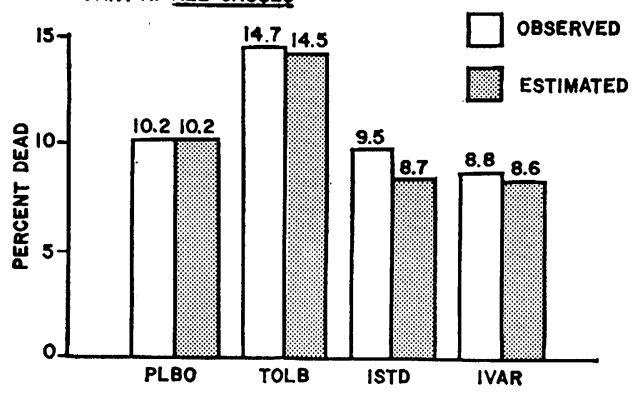

PART B. CV CAUSES

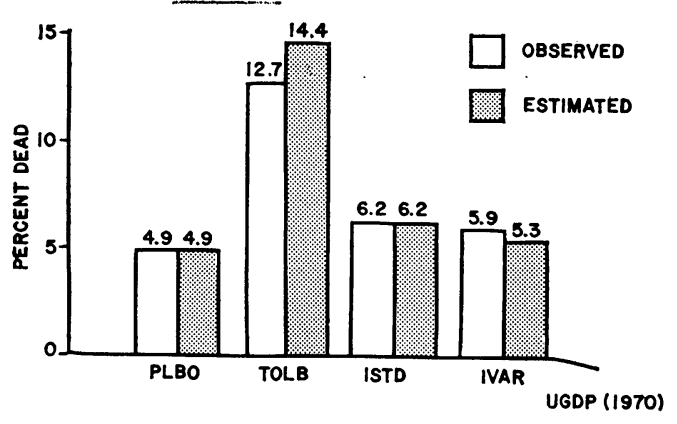

Figure 11

CUMULATIVE MORTALITY RATES PER 100 POPULATION AT RISK

BY YEAR OF FOLLOW-UP UGDP, JANUARY 1971
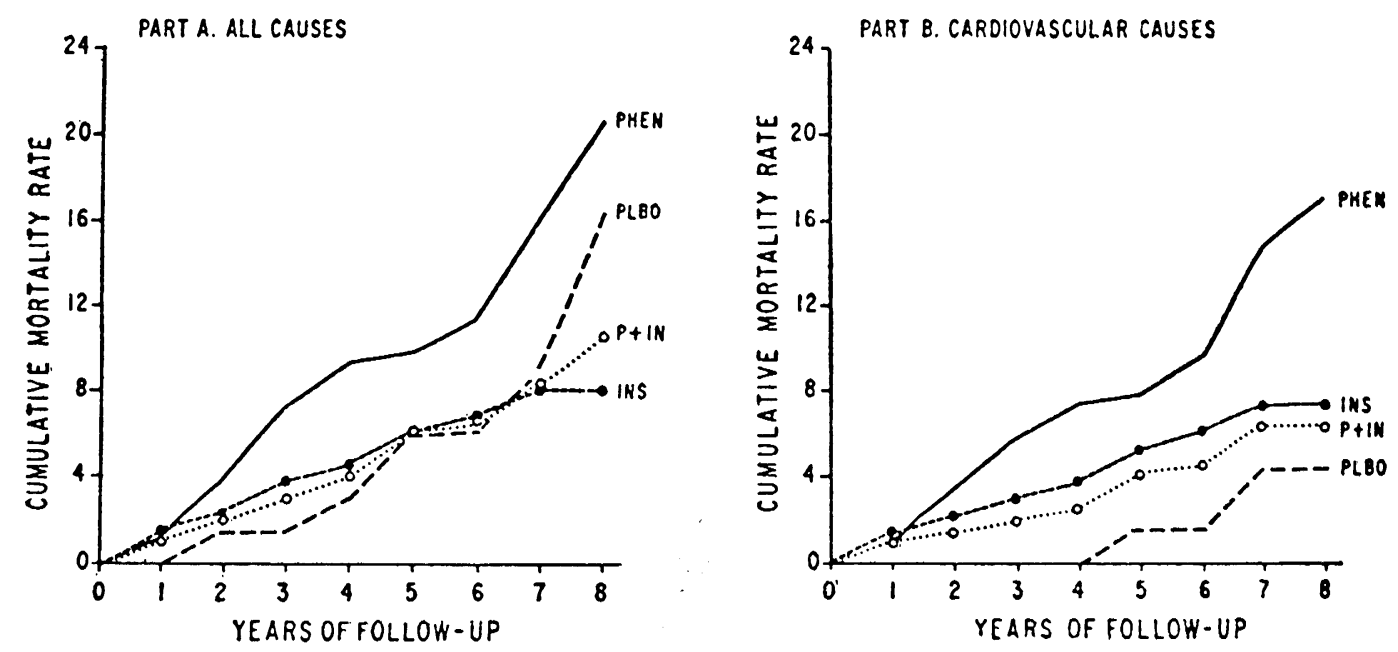

$\operatorname{UGDP}(|97|)$ 
blood pressure levels and a greater proportion died with new hypertension than in all other treatment groups ${ }^{10)}$.

\section{EXPERIENCE IN THE CDP}

Another difficult problem in the analysis of data and in the decision making process is illustrated by our CDP experience. In the CDP we have six treatment groups including a placebo control group (Figure 12) ${ }^{13)}$. Four active cholesterol lowering drugs have been tested

Figure 12

\begin{tabular}{|c|c|c|}
\hline \multicolumn{3}{|c|}{$\begin{array}{l}\text { TREATMENTS AND NUMBER OF PATIENTS ASSIGNED } \\
\text { TO EACH TREATMENT IN THE CDP }\end{array}$} \\
\hline TREATMENT (ABBREVIATION) & DOSAGE PER DAY & NO. OF PATIENTS \\
\hline ESTROGEN-LOW DOSE (ESG1) & $2.5 \mathrm{mg}$ & 1,101 \\
\hline ESTROGEN-HIGH DOSE (ESG2) & $5.0 \mathrm{mg}$ & 1,119 \\
\hline CLOFIBRATE (CPIB) & $1.8 \mathrm{gm}$ & 1,103 \\
\hline DEXTROTHYROXIN (D-T4) & $6.0 \mathrm{mg}$ & 1,110 \\
\hline NICOTINIC ACID (NICA) & $3.0 \mathrm{gm}$ & 1,119 \\
\hline \multirow[t]{2}{*}{ PLACEBO-LACTOSE (PLBO) } & $3.8 \mathrm{gm}$ & 2,789 \\
\hline & TOTAL & 8,341 \\
\hline
\end{tabular}

in the CDP: estrogen (low dose and high dose), clofibrate (CPIB), dextrothproxin (D-T4), and nicotinic acid. The CDP is also a randomized double-blind long-term clinical. The background, design, methods, and baseline findings of the CDP have been published as a supplement to the journal CIRCULATION ${ }^{5)}$.

The CDP data have been reviewed extensively every six months and briefly every three months by the CDP Data and Safety Monitoring Committee.

As of August 1971, the D-T 4 group showed a higher total mortality compared to the placebo group ${ }^{7)}$. The difference in overall mortality between the two treatments was of borderline significance. In the middle section of Figure 13 mortalities are given by CDP risk group. Risk group 1 comprises the patients with one uncomplicated previous myocardial infarction, while risk group 2 comprises the patients with one complicated myocardial infarction or multiple myocardial infarctions. An excess mortality is observed only in risk group 2 patients. The right section of Figure 13 shows mortalities for subgroups based on history of angina pectoris and heart rate at entry. D-T 4-treated patients showed an excess mortality in the subgroup of patients with a history of angina pectoris and heart rate of 70 or greater per minute, wihle in another subgroup of patients with no history of angina pectoris and heart rate less than 70 per minute D-T4-treated patients showed a significantly lower mortality than placebo-treated patients. Other subgroups of patients showed about the same mortalities. Figure 13 illustrates the situation where the effect of drugs may differ among subgroups or may be we are observing differences due to chance alone. 
Figure 13

PERCENT DEATHS BY SELECTED BASELINE CHARACTERISTICS

IN THE D-T 4 AED PLBO TREATMENTS IN THE CDP

AUGUST 1, 1971

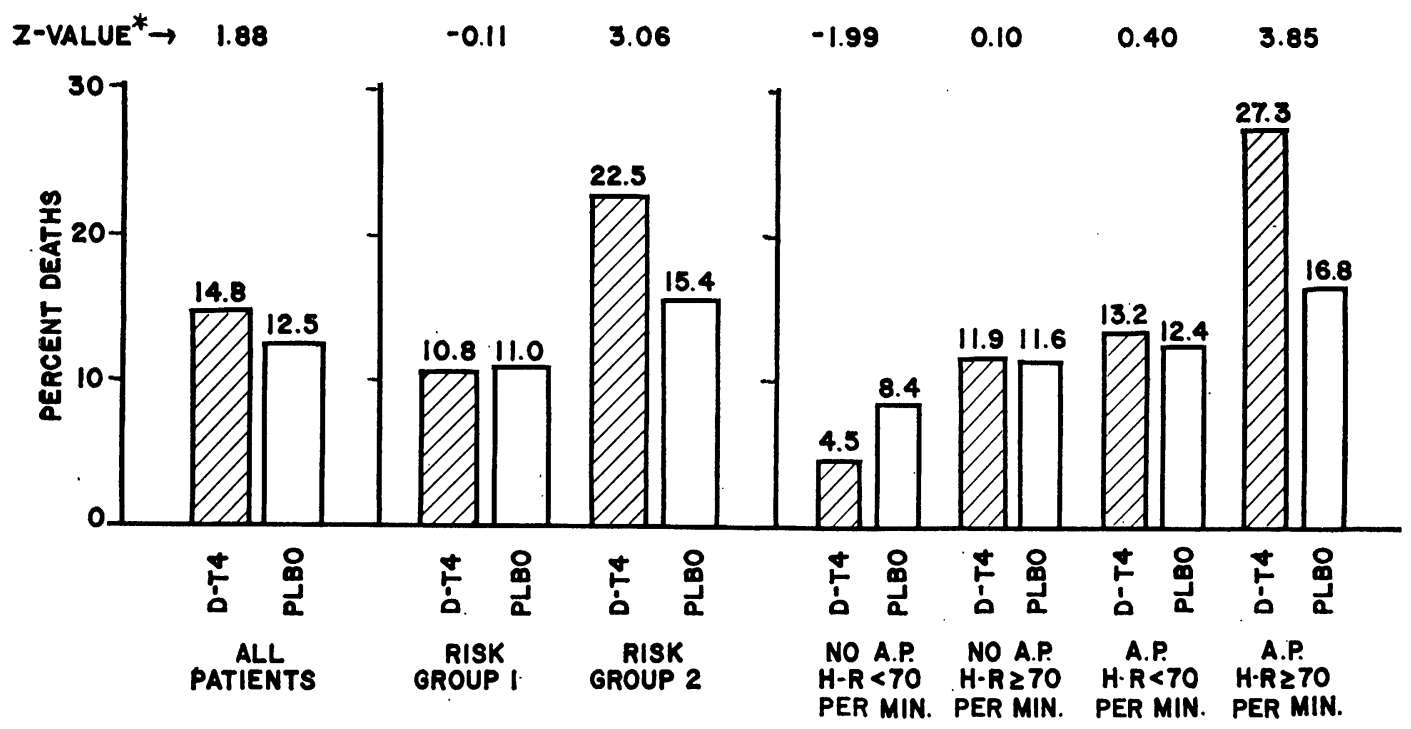

*(D-T4-PLBO)/S.E. OF DIFFERENCE

$\operatorname{CDP}(1971)$

This type of apparently disparate subgroup findings posed the major problem which had to be weighed by the study leadership in arriving at the decision concerning discontinuation of medication for patients receiving dextrothyroxine ${ }^{1)}$. The first and key question was the following: Is the apparent marked excess in mortality for certain D-T 4 subgroups a valid phenomenon, warranting a decision to stop medication for these subgroups? In view of the method for identifying these subgroups, this question was not easy to answer. That is, at the outset of the study, no hypothesis had been formulated concerning possible toxicity of D-T 4 in certain subgroups identified by baseline characteristics. The subgroups with excess mortality were selected during the course of the project, in part based on medical judgment from the limited experience of earlier studies, and in part based on computer searches. Under these circumstances, classical tests of statistical significance and usual criteria of significance cannot be properly utilized to assess the possibility that observed differences can arise by chance. To cope with this problem a Monte Carlo simulation technique was used to test the possibility that the excess mortality for selected D-T 4 subgroups could be a chance phenomenon. The descriptions of detailed method of this technique and results are beyond the scope of this paper. They were described in one of the CDP reports ${ }^{7}$.

The conclusion from this analysis is that the size of the excess mortality in D-T4 subgroups was definitely larger that what would be expected as a result of the computer selection process based on actual vs. random assignment of mortality status for D-T 4 and placebo patients. Therefore, the observed excess mortality for selected D-T 4 subgroups was 
very likely a real, not a chance phenomenon.

After further data evaluation the CDP Data and Safety Monitoring Committee recommended to discontinue the D-T 4 treatment.

\section{COST AND SPONSOR OF LARGE-SCALE CLINICAL TRIALS}

The final part of this presentation will be devoted to a very difierent set of problems. It has to do with the appropriateness of financing from the public sector versus the private, profit-making sector. In many instances, development of new treatments cannot expect to be rewarding from a point of view of industry. The number of patients with parkinsonism suitable for treatment with L-dopa is too small to recoup development costs. Many other conditions could be quoted in which there could be no profit motive involved. These are appropriate areas for the public sector to undertake financial responsibility for. In othor areas, however, such as primary or secondary coronary infarction prophylaxis, the market potentially is huge; and it could be considered appropriate for industry which might benefit from a positive result to undertake the risk of supporting such a trial. Indeed the Food and Drug Administration requires positive proof of efficacy in addition to a corresponding absence of serious side reactions. In other words, the benefit/risk equation ought to be solved in favor of benefits. In a sense this requirement is difficult for industry to meet since not only is it costly but industry in general does not have direct access in a numerical sense to the patient material required. Also, there is the need for an unbiased management and supervisory body so that the study's findings can be accepted without having the particular vested interest in mind. Thus for such studies there is a need of an agency of public standing which will sponsor such studies, which will appoint groups of people uninvolved in material interest, which will supervise and make policy decisions, and shoulder the ethical point of view of either breaking off a study or continuing in the face of as yet nonsignificant trends. The FDA is not necessarily suitable for such a repository since it would have to be judge, jury, and defendent at the same time. As it has regulatory obligations it could not very well also sponsor the crucial studies in man. We therefore need a new agency which deals with the sponsorship of such studies and is capable of disbursing the funds received from industry and of monitoring large-scale clinical trials of the phase IV type which I have described; namely, studies where clinical benefit and clinical risk are the crucial points and not a chemical response assumed to be helpful such as the lowering of blood glucose levels.

The importance of conducting such phase IV type clinical trials are obvious in spite of the great amount of time and money required. The National Heart and Lung Institute alone is currently engaged in supporting at least five major clinical trials. One deals with enzymes, urokinase, and streptokinase which may dissolve thrombi at the point of formation and thus help patients with pulmonary emboli, myocardial infarction, and stroke. Another one is involved with primary prophylaxis of coronary artery disease by simultaneously intervening with hypertension, hypercholesterolemia, and cigarette smoking habit. A third study attempts to compare a rigorously supervised management of hypertension versus a loose 
one such as the general practitioner may apply. Then there is a study dividing the population into blood lipid patterns according to the Frederickson lipoprotein type and superimposing clinical trials with various cholesterol-lowering regimens as primary prophylactics: cholestyramine, colestipol resins, or clofibrate. Lastly, the national Coronary Drug Project ${ }^{5}$-perhaps the world's largest long-term clinical drug trial. An offshoot of the latter began recently; namely, a controlled clinical trial of aspirin as a secondary prophylactic in postcoronary patients.

The National Cancer Institute has pioneered in the area of drug trials, and its chemotherapy program is still flourishing. Currently more than a dozen such groups are active; many have a large number of protocols on which they are working. Not all of these studies fall in the category of cooperative clinical trial of the phase IV type where a clinical response is the final endpoint.

Finally, it is sincerely hoped that many large-scale clinical trials, hopefully phase IV type, will be conducted in Japan or cooperatively between Japan the U.S. in the future.

\section{REFERENCES}

1) University Group Diabetes Program. A study of the effects of hypoglycemic agents on vascular complications in patients with adult-onset diabetes. I. Design, method and baseline characteristics and II. Mortality results. Diabetes, 19 (Suppl 2) : 748-830 (1970).

2) University Group Diabetes Program. Effects of hypoglycemic agents on vascular complications. in patients with adult-onset diabetes. III. Clinical implications of UGDP results. JAMA, 218 : 1400-1410 (1971).

3) University Group Diabetes Program. Effects of hypoglycemic agents on vascular complications. in patients with adult-onset diabetes. IV. A preliminary report on phenformin results. JAMA, 217 : 777-783 (1971).

4) Cornfield, J., The University Group Diabetes Program. A further statistical analysis of the mortality finding. JAMA, $217:$ 1676-1687 (1971).

5) Coronary Drug Project Research Group. The Coronary Drug Project. Design, methods and baseline results. Circulation, Vol. 47-48; No. 3, Suppl I (1973).

6) Coronary Drug Project Research Group. The Coronary Drug Project. Initial findings leading to modifications of its reseach protocol. JAMA, $214: 1303-1313$ (1970).

7) Coronary Drug Project Research Group. The Coronary Drug Project. Findings leading to further modifications of its protocol with respect to dextrothyroxine. JAMA, 220:996-1008 (1972).

8) Cornfield, J. The bayesian outlook and its application. Biometrics, $25: 617-657$ (1969).

9) Canner, P. Monitoring treatment differences in long-term clinical trials. Submitted to J. Amer. Stat. Assoc. (1973).

10) University Group Diabetes Program. A study of the effects of hypoglycemic agents on vascular complications in patients with adult-onset diabetes. V. Evaluation of phenformin therapy. Diabetes (1974). in press.

11) Lasseter, K. D., Tevey, G. S., Palmer, R. F., et al. The effects of sulfonylurea drugs on rabbit myocardial contractility, canine purkinje fiber automaticity, and adenyl cyclic activity from rabbit and human hearts. J. Clin. Invest. 51 : 2429-2434 (1972).

12) Jick, H. and Slone, D. Personal communication. 